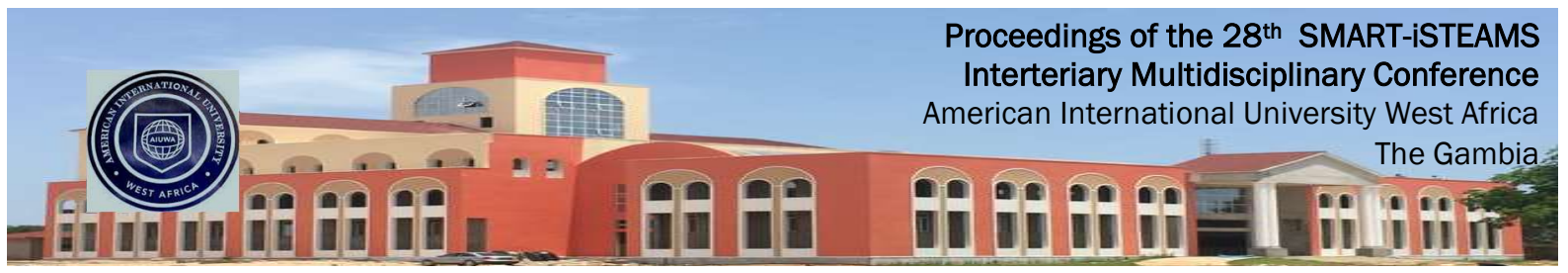

\author{
Full Research Paper
}

\title{
Real-Time Nitrogen Dioxide Pollutant Monitoring In Lagos State, Nigeria Using Wireless Sensor Networks
}

\section{Kelvin.O.Ovabor Simeon.T.Apeh}

Dept of Computer Engineering University of Benin

Benin City

Nigeria

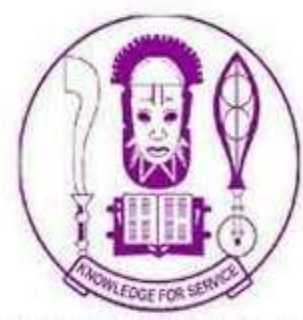

E-mails

1ovabor.k@nafdac.gov.ng

2apeh@uniben.edu

Phones:

$1+2347030380057$

$2+2348034956812$

\begin{abstract}
Wireless Sensor Networks (WSNs) find its application in various facets of life. Atmospheric contamination in the various elements of air leading to hazardous effects of global warming and acid rains can affect the well-being of a population. An air pollution monitoring system is therefore important to keep away from such adverse imbalance in nature. In the proposed paper, an attempt is made to develop a real time pollution monitoring using wireless sensor networks (WSN). This WSN will monitor the profile concentration of nitrogen dioxide (NO2) in a chosen location. With the rapid growth in the industries, which are the main sources of air pollutants, the problem of air pollution is becoming a serious concern for the health of the population. The concentration of one major air pollutant gases Nitrogen dioxide (NO2) from the air is sensed by the gas sensors. The sensor is properly calibrated as per the standard methods and the gas sensor is then incorporated with the wireless sensor motes using hierarchical cluster-based architecture. The node is expected to sense pollutants, convert, and process the magnitude of pollution to equivalent data output which is transmitted to the remote base station. The expected results would show data of the Nitrogen dioxide sensor for each location and graphical results. Research and suggestions have been offered by researchers for two decades and more on the increasing dangers of industrialization, population growth, influx of used vehicles popularly called 'tokunbos' as they pertain to the public health and adverse effects if not properly managed. The test findings of this work still show if the concentration of gaseous air pollutant like NO2 in a chosen location and at a particular time poses a problem to humans and the environment.
\end{abstract}

Keywords-Air pollution, Wireless sensor networks, Nitrogen dioxide, Environmental risk, public health, Hierarchical cluster-based architecture

Proceedings Reference Format

Ovabor, K.O \& Apeh, S.T. (2021): Real-Time Nitrogen Dioxide Pollutant Monitoring In Lagos State, Nigeria Using Wireless Sensor Networks. Proceedings of the 28th iSTEAMS Intertertiary Multidisciplinary Conference. American International University West Africa, The Gambia. October, 2021. Pp93-104 www.isteams.net/gambia2021. DOI - https://doi.org/ 10.22624/AIMS/iSTEAMS2021/V28P7 


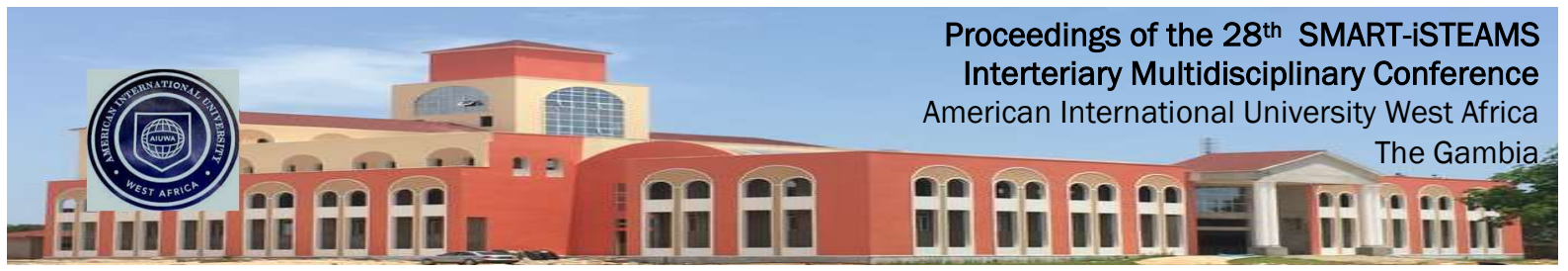

\section{INTRODUCTION}

Wireless Sensor Network (WSN is a fast-evolving technology area that is gaining importance due to its various applications. It is an intense wireless network of small, low-priced detectors, which collect and distribute environmental data. With the use of WSNs, supervision and control of physical environments from remote locations can be acted with improved accuracies and timeliness [1]. Air pollution is a serious global problem because it presents a major environmental risk to health of a population. This problem is compounded by the rapid growth of urban population, industrial development, and the increase of the transportation activities [2]. The main cause of air pollution is the burning of fossil fuels. Meanwhile, the consumption of fossil fuels continues to outweigh the use of more environmentally friendly forms of energy. This condition inadvertently has had a major effect on air quality [3].

Transportation of motor vehicles is the main source of air pollution in urban areas. Emissions generated by motorized vehicle activities in urban areas were mainly NO2 gas originating from the combustion process [4],[5]. The impact of the use of fossil fuels clearly will reduce air quality. Furthermore, declining air quality will cause serious impacts on human health. According to [6], air pollution is an important risk factor that can lead to mortality and morbidities. It was estimated that half of the deaths that occurred were caused by heart disease, stroke and lung cancer which could be attributed to air pollution. The exposure to air pollution from both outdoor and household (indoor) resulted in the deaths of around 7 million people annually in all regions of the world, while Western Pacific and Southeast Asia were the most affected [6]. WHO estimated that there were 200 thousand deaths due to outdoor pollution that mainly occur in urban areas, where about $93 \%$ of cases occurred in developing countries [7].

Air pollutants could come from anthropogenic activities including industry, transportation, offices, and housing, but could also originated from natural activities. The transportation and industrial sectors were the highest contributors to the declining of air quality. It has been predicted that air pollution due to transportation and industrial activities will increase 10-fold in 2020 compared to the conditions in 1990 [8]. Instruments usually used for atmosphere pollution monitoring are Fourier Transform Infrared (FTIR), mass spectrometers and gas chromatographs which give precise and selective gas readings. Because of the bulk size of the instruments, there is high cost of maintenance for a larger scale monitoring application [9].

Gas sensors are becoming equally effective alternative [10] as they are low cost, compact and robust with versatile applications. Gas sensor monitoring methods are basically electrochemical, catalytic bead, photo ionization, infrared and solid-state [9]. Present monitoring methods mainly employ smart transducer interface module (STIM) with semiconductor gas sensors. Geosensor network with control action for monitoring [11] are also unfavorable due to high cost. In this paper, an attempt is made to propose and design a system which is economical, consistent, precise and scalable for the real-time pollution monitoring in an industrial city like Lagos using with WSN. Hierarchical cluster-based architecture is best suited for habitat and environmental monitoring applications with its routing schemes. A hierarchical topology was adopted for this work because a wide geographical coverage area is part of the goal of the WSN design. 


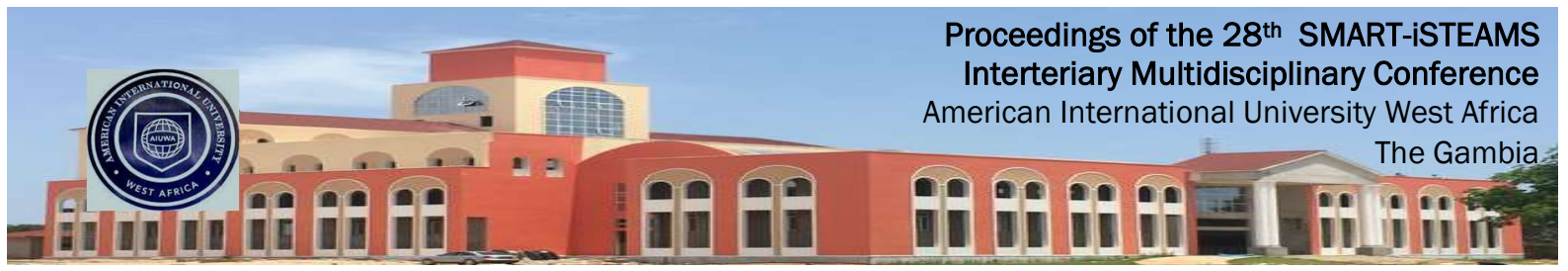

\section{HIERARCHICAL CLUSTER-BASED ARCHITECTURE}

Hierarchical cluster-based architecture is best suited for habitat and environmental monitoring applications with its routing schemes. Hierarchical cluster-based routing schemes are based on the fact that the energy consumed to send a message to a distant node is far greater than the energy needed for a short-range transmission and that this will contribute longer lifespan [12]. In the hierarchical model adopted in this work, sensor nodes are aggregated to form clusters based on their power levels and proximity. Each cluster has a cluster head also called coordinator in this context, has the task of acquiring data of the sensor nodes in its cluster. In other words, a zone could have several clusters and each cluster have a cluster head or coordinator and each coordinator has some sensor nodes. This kind of setup aid in scalability of WSNs [12].

This type of WSN architecture tagged a 3-level hierarchical cluster-based network. It is very useful for regionally managing sensor networks. When a user's query is disseminated to the network through the sensor gateway and the sink node, the responses received from the correspondent sensor nodes are first aggregated to the coordinators, and then each area coordinator aggregates the response data from the cluster heads in the area, finally all the areas having the response data send the aggregated result to the gateway through the sink node. Accordingly, the gateway can acquire the regional sensing data corresponding to the user query from each area node [12].

The hierarchy network system based on the use of clusters was adopted based on the advantages it offers in terms of energy efficiency, coverage distance. The entire network was set up to employ a three-layered hierarchy cluster-based design which is gateway, clusters head and sensor nodes. One of the nodes of a cluster was selected as the cluster-head referred to as coordinator, which acts as the gateway of that cluster to the main network, and it is responsible for acquiring data of the sensor nodes in its cluster. The clusters can only communicate with their cluster heads or coordinators like in a star topology [12].

The cluster-heads and the gateways are setup in mesh topologies as they may communicate with other coordinators (of other clusters) or may directly transmit data to a particular gateway. The coordinators were setup to perform most of the data collection, aggregation and processing to minimize the redundancy of the information collected by the sensor nodes of the cluster leaving the gateways to serve as gateways to the sink which is the base station. A zone consists of gateway and several cluster heads. That is, the entire sensor network has some zones, a zone has some clusters with cluster heads, and a cluster head has some sensor nodes. This kind of structure, i.e., a 3-level hierarchical cluster-based network, is very useful to regionally manage the sensor network. 

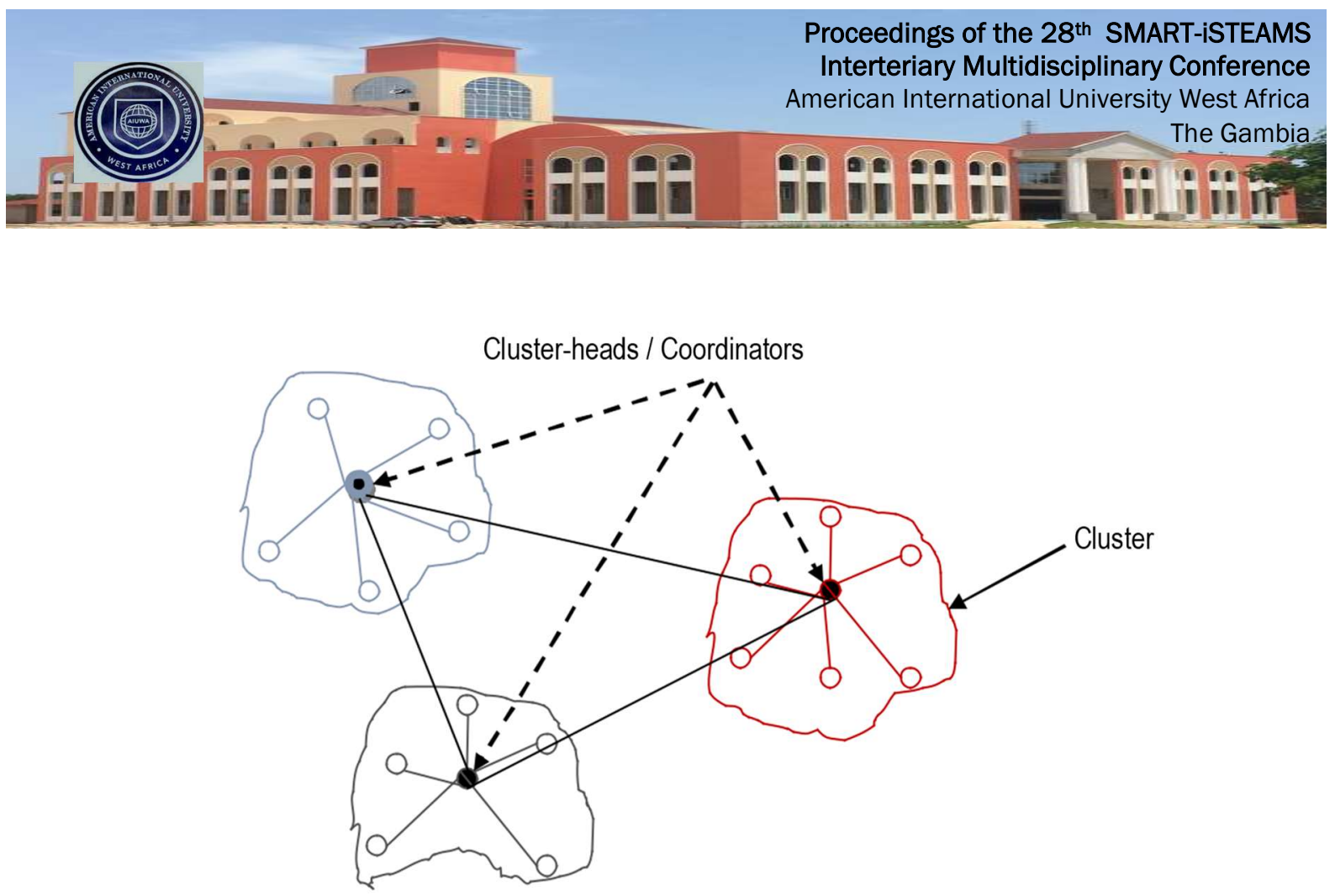

Fig 1. Sensor Nodes organized into clusters with cluster-heads acting as gateway for the nodes that belong to their cluster

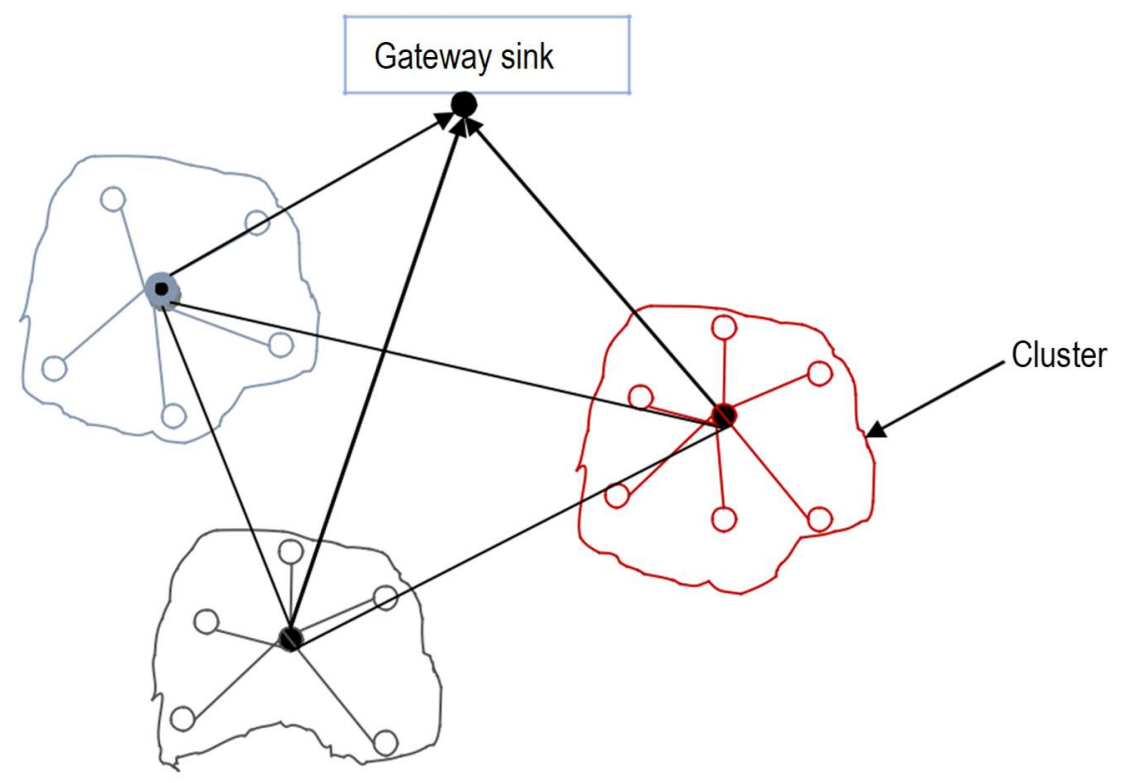

Fig 2. Example of a network with LEACH 


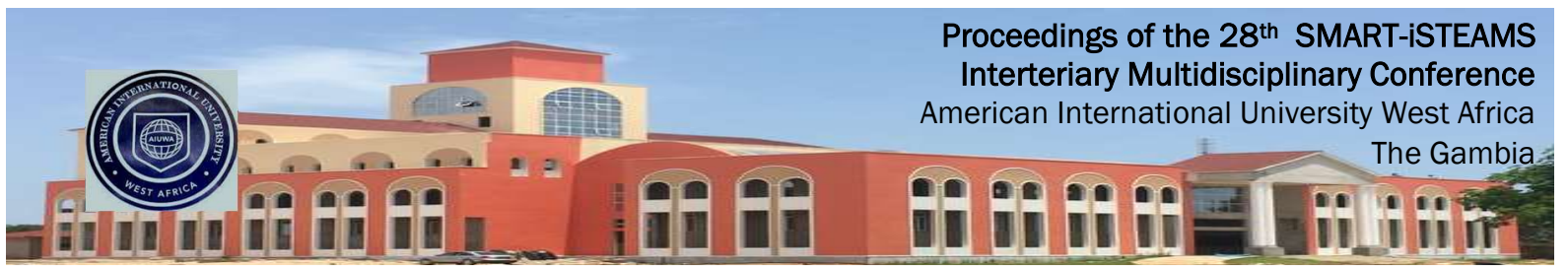

The cluster-heads receive data from the nodes of their clusters, and after being processed, the data are transmitted by the cluster-heads directly to the gateway-sink.

\section{STUDY AREA}

The foremost industrial states in Nigeria are Lagos, Rivers, Kano and Kaduna states with Lagos having the largest population density of them all. Lagos state also has the highest concentration of industries, with well over seven thousand medium and large-scale industrial establishments [13]. There is a claim that about $70-80 \%$ of the manufacturing facilities operating within the medium and large-scale industries are located there in lagos. The major industrial estates in Lagos are: Ikeja, Agidingbi, Amuwo Odofin (industrial), Apapa, Gbagada, Iganmu, ljora, Ilupeju, Matori, Ogba, Oregun, Oshodi/Isolo/Ilasamaja, Surulere (light industrial) and Yaba [14,15,16].

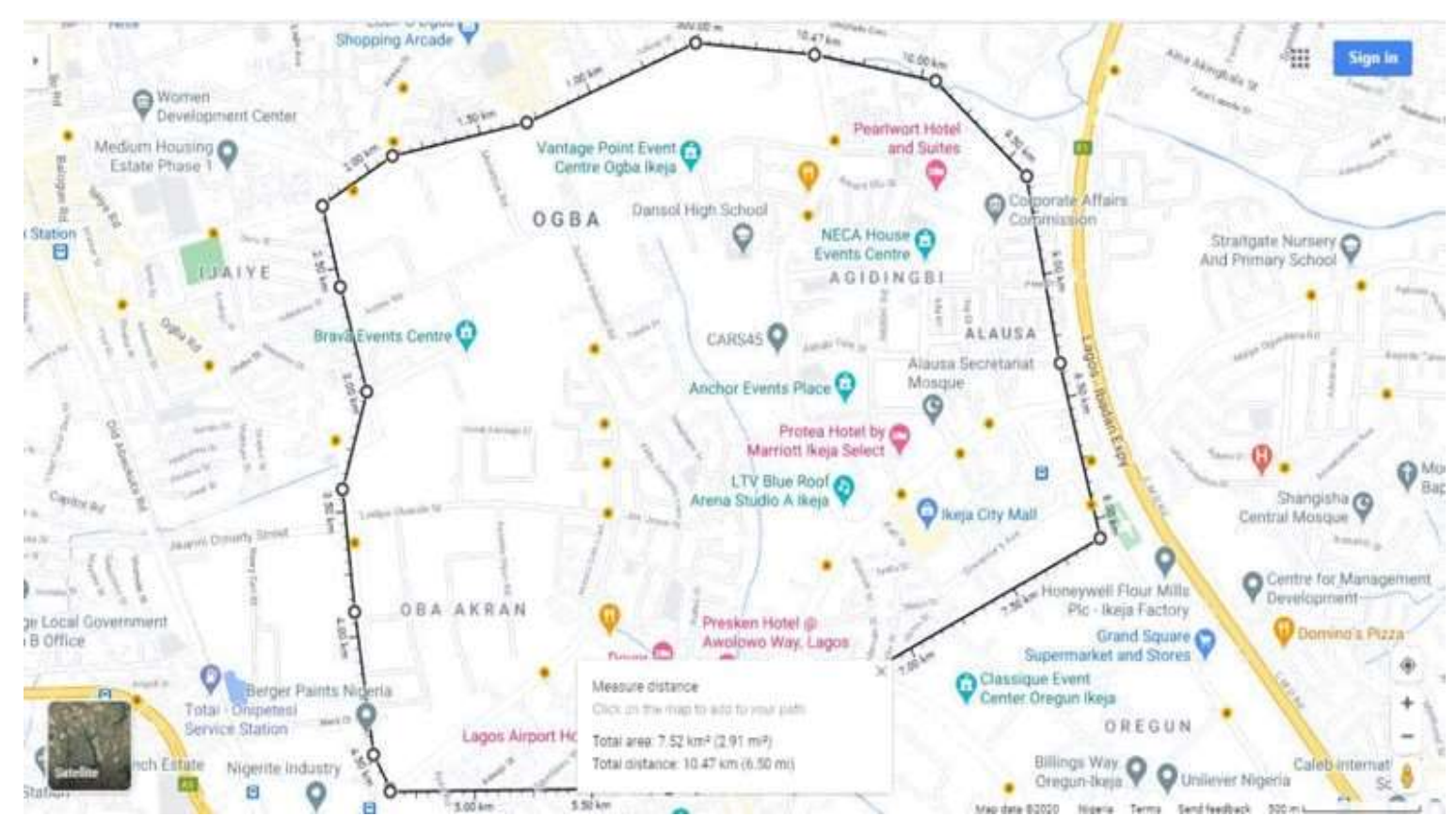

Fig 3. Map of the Ikeja industrial Estate Study Area

The study area as shown on the Google map has a total area of $7.52 \mathrm{~km} 2(2.51 \mathrm{mi} 2)$ and a total distance of $10.47 \mathrm{~km}$ and its GPS coordinates are 6.63026, 3.34569 north, 6.2011 , 3.36136 East, 6.61594, 3.33544 West, 6.0016, 3.34668 South. 

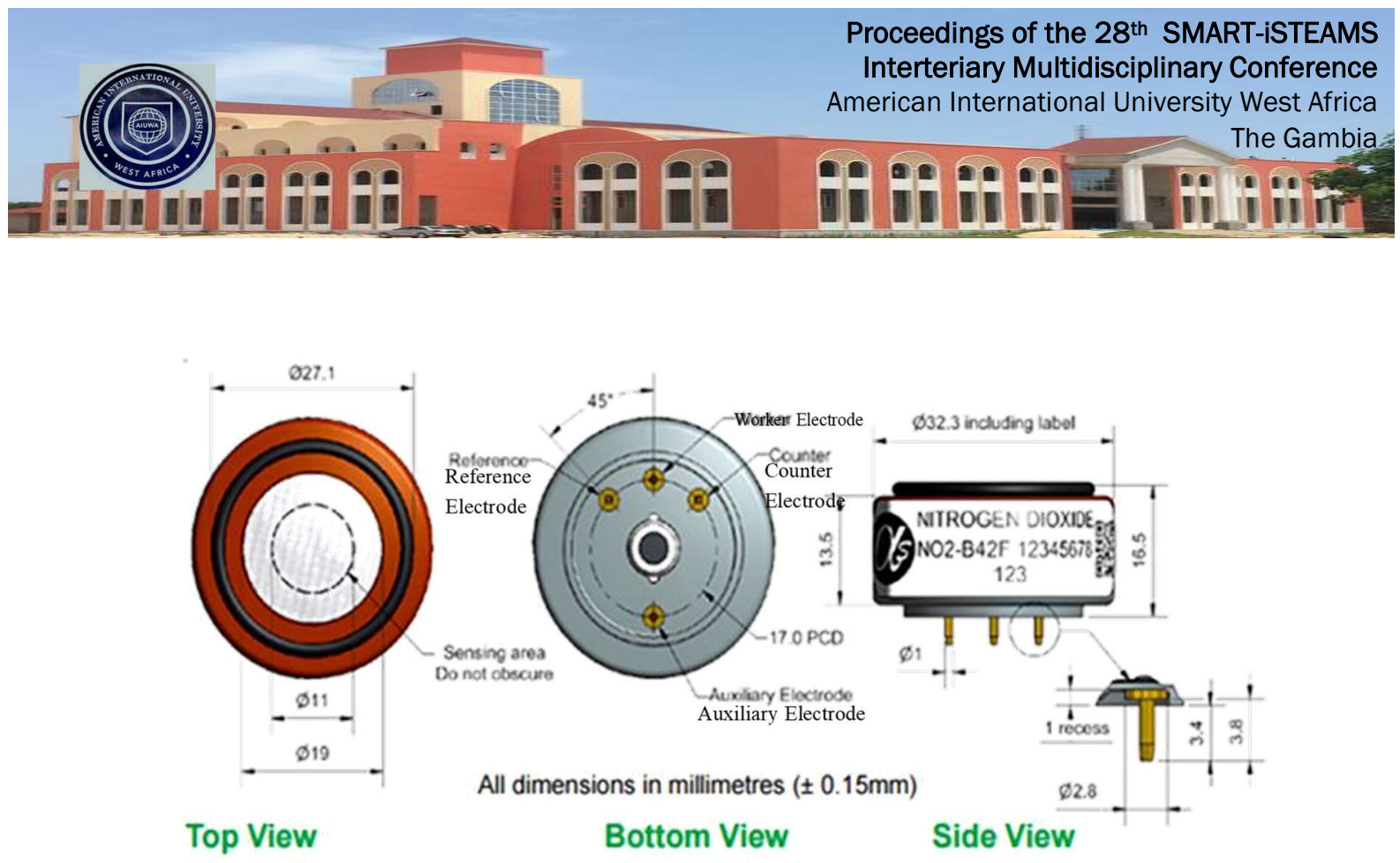

Fig 4. Different views of the Nitrogen Dioxide sensor

\section{NITROGEN DIOXIDE SENSOR}

The Alphasense NO2-B42F sensor is used to measure ambient NO2 in many low-cost air quality settings. The Alphasense B4 electrochemical gas sensing series used employs a four-electrode approach. The electrodes are embedded in an electrolyte solution separated from the atmosphere by a semi-permeable membrane. The gas of interest diffuses through the membrane into the electrolyte where it contacts a "working" electrode and is either oxidized (in the case of $\mathrm{NO}$ and $\mathrm{CO}$ ) or reduced (NO2 and 03 ).

The potential at the working electrode is maintained at a constant value with respect to a "reference" electrode. Electric charge produced at the working electrode is balanced by the complementary redox reaction at a "counter" electrode, generating an electric current. The sensor also contains an "auxiliary" electrode, which shares the working electrode's catalyst structure, but is isolated from the ambient environment, accounting for fluctuations in the background current associated with other processes at the electrode and electrolyte. Subtracting the auxiliary current from the working current gives a corrected current dependent on the gas concentration [17]. The humidity dependence is included in the temperature dependence, as there is no evidence for independent humidity dependence and relative humidity exhibits an anti-correlation with temperature in the field.

NO2-B42F Nitrogen Dioxide Sensor Specifications:

(1) Range(ppm): 0 20

(2) Sensitivity: $-160 \sim 320 \mathrm{nA} / \mathrm{ppm}$ at $2 \mathrm{ppm} \mathrm{NO} 2$

(3) Response time: t90 <70(s) from zero to $2 \mathrm{ppm} \mathrm{NO2}$

(4) Zero current: $-25 \sim 50 \mathrm{nA}$ in zero air at $20^{\circ} \mathrm{C}$

(5) Operating life: $>2$ years

(6) Size $(\mathrm{mm})$ : $\Phi 32.0 \times 16.5(\mathrm{~mm})$

(7) Linearity: linear at zero and 5ppm NO2; $< \pm 1 \mathrm{ppb}$ error at full scale 


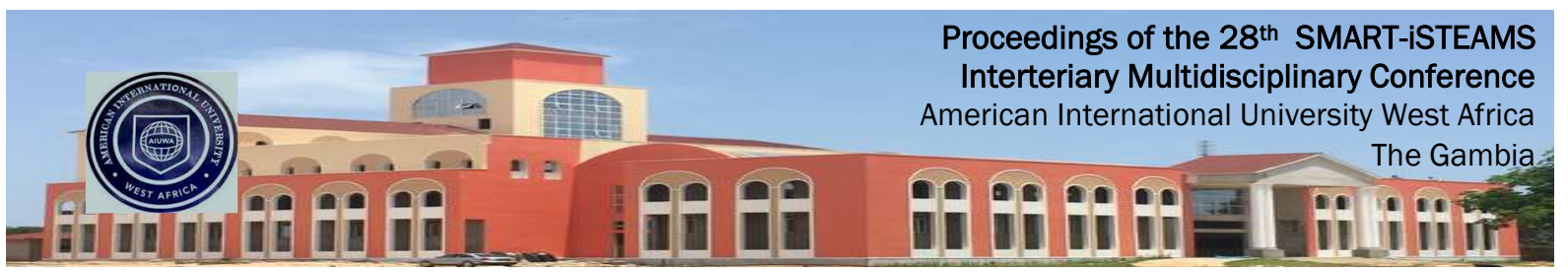

(8) Over gas limit: 50ppm

(9) Pressure range: $80 \sim 120 \mathrm{kPa}$

(10) Weight: < 13g

(11) Temperature range: $-30 \sim 40^{\circ} \mathrm{C}$

(12) Humidity range: $15 \sim 85 \% \mathrm{RH}$

(13) Load resistor: 33 100 $\Omega$

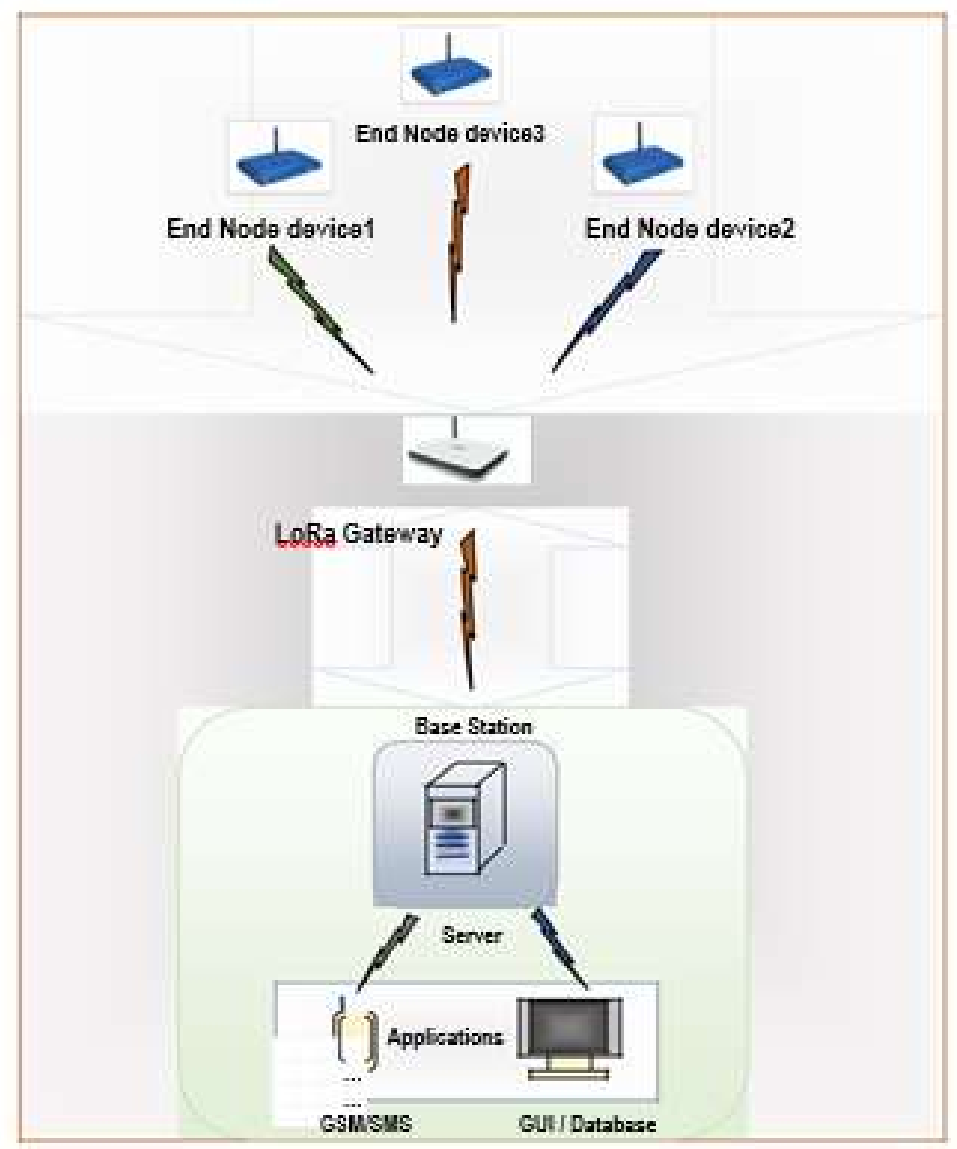

Fig 5. Wireless Sensor Network (WSN) based Air Pollution and Reporting System Architecture

\section{WSN BASED NO2 POLLUTION MONITORING AND REPORTING SYSTEM ARCHITECTURE}

Fig.5 depicts the wireless sensor network (WSN) application in air pollution monitoring and reporting architecture and concept as embarked on this study. It shows the node of the sensor and a gateway. The sensor nodes or end devices send their sensor data to the gateway they are tuned to through necessary configuration settings. The gateway eventually collates all sensor node data after receiving data from the nodes and sends it to the base station. 


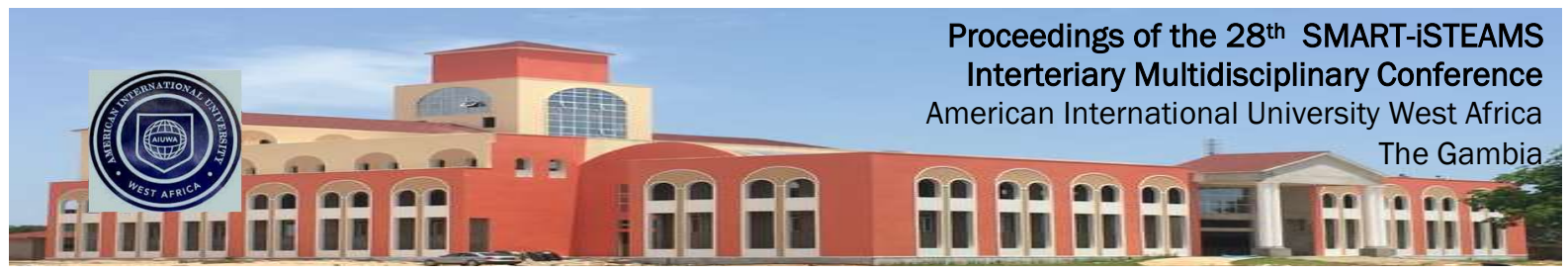

The remote base station also referred to the computer systems that has is serving as the server or data hub. The server has installed software with developed applications to handle database and data presentation through graphic user interface (GUI) for human machine interaction (HMI). The applications in the server stores information of the network (WSN) sensor nodes, sensor locations, time, and date of transmissions for data reception.

The major functional blocks of the system are listed as;

1. Sensor Nodes

2. Gateway

3. Remote Base Station (Server)

TABLE 1: RESULTS FOR ZONE 1 FOR A TYPICAL WARM DAY WITH AVERAGE TEMPERATURE OF $280 \mathrm{C}$ AND HUMIDITY OF $65 \%$.

\begin{tabular}{|c|c|c|c|}
\hline Time & NO2 & Temperature & Humidity \\
\hline 7.09 & 0.072 & 26 & 71 \\
\hline 8.10 & 0.084 & 27 & 69 \\
\hline 9.09 & 0.09 & 27 & 70 \\
\hline 10.09 & 0.086 & 28 & 67 \\
\hline 11.10 & 0.089 & 29 & 63 \\
\hline 12.09 & 0.085 & 30 & 62 \\
\hline 13.09 & 0.083 & 31 & 60 \\
\hline 14.09 & 0.087 & 32 & 59 \\
\hline 15.10 & 0.090 & 33 & 57 \\
\hline 16.09 & 0.095 & 33 & 54 \\
\hline 17.10 & 0.096 & 30 & 58 \\
\hline 18.09 & 0.092 & 29 & 62 \\
\hline 19.09 & 0.083 & 27 & 64 \\
\hline 20.10 & 0.076 & 27 & 66 \\
\hline 21.09 & 0.071 & 27 & 67 \\
\hline 22.09 & 0.049 & 27 & 70 \\
\hline 23.09 & 0.036 & 26 & 73 \\
\hline 0.09 & 0.026 & 26 & 73 \\
\hline 1.10 & 0.025 & 26 & 74 \\
\hline 2.09 & 0.018 & 25 & 76 \\
\hline 3.09 & 0.015 & 25 & 77 \\
\hline 4.09 & 0.021 & 25 & 78 \\
\hline 5.09 & 0.028 & 25 & 76 \\
\hline 6.10 & 0.046 & 26 & 72 \\
\hline Average & 0.064 & 27 & 65 \\
\hline
\end{tabular}




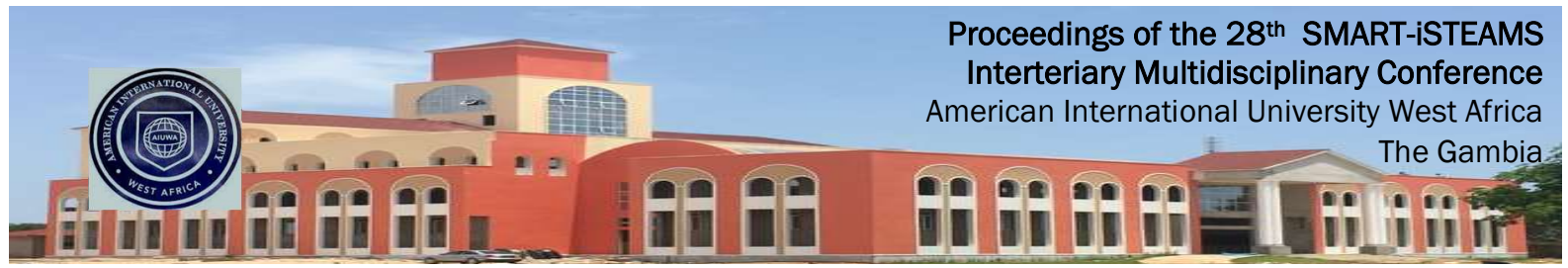

Basically, real-time ambient air pollutant concentrations during measurements always have random spatial behavior that affects interpretation of data hence the data presented were for every one-hour as programmed. The extra 9 to 10 seconds were for the gas sensor pre-heating time as the main code starts the process on the hour.

Figure 6 shows the variation of NO2 gas pollutants for 24 hour/daily readings. These readings indicate that the average emissions NO2 is relatively higher during peak periods. This is mainly because its main emission sources are of vehicular origin. The readings show that the late evenings (night-time) to early mornings have low readings obviously due to lesser vehicular movements and activities and this time range falls between $11 \mathrm{pm}$ to $5 \mathrm{am}$. The higher concentrations are more in the mornings through afternoon to early evenings when students and workers are on the move to work and school thus increasing density of vehicular activities and movement. It is slightly lower at about 12 noon to $2 \mathrm{pm}$ owing to the less presence of workers and students from school and work. But the general economic activities that are high in these zones still results in high pollution levels as shown by the readings. The following charts show a typical daily reading for a warm day that is not very sunny.

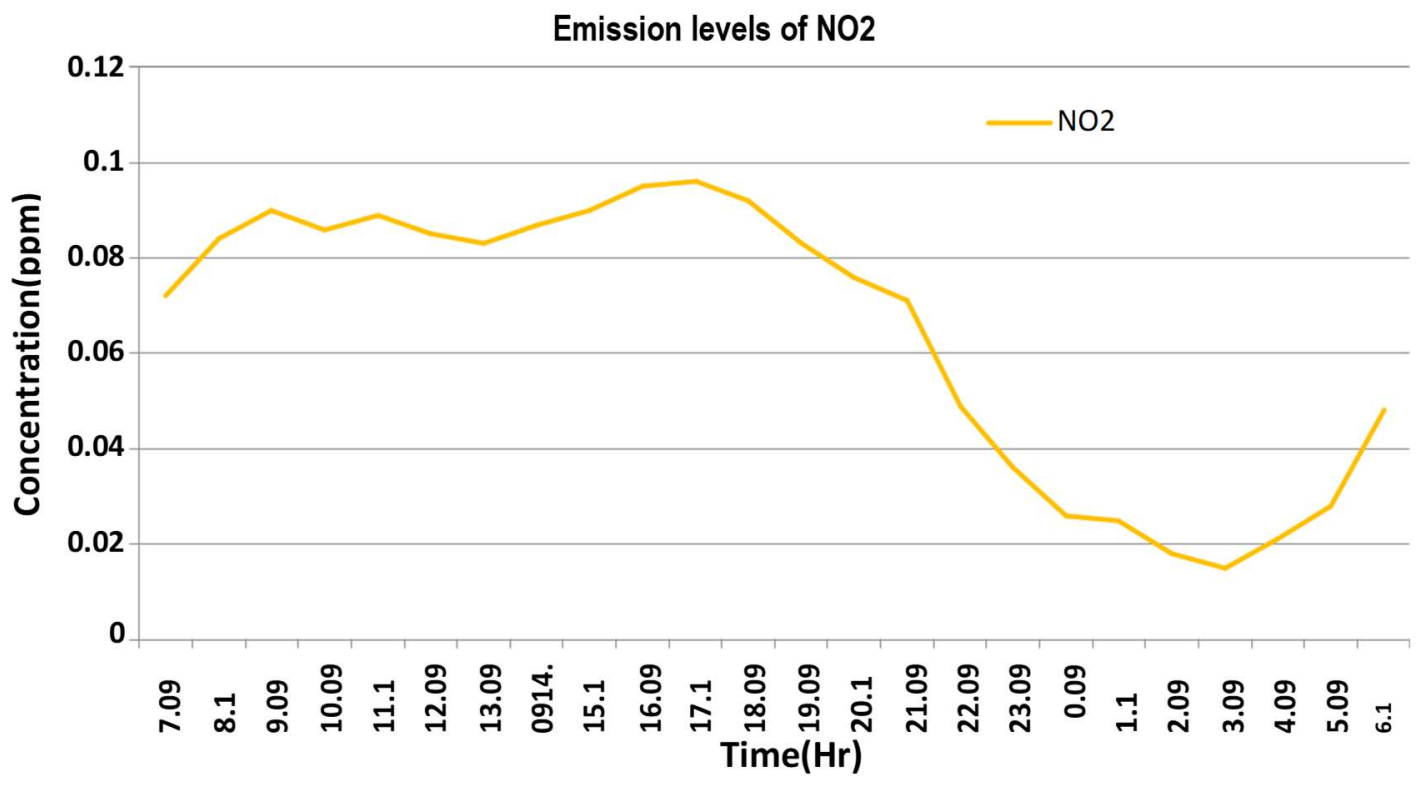

Fig 6. Chart of typical daily variation of Nitrogen Dioxide

The chart for NO2 shows that the average exceeds WHO and FEPA limit of $0.06 \mathrm{ppm}$ slightly for a 24hour period. The readings also show strong NO2 presence during the morning hours to evening hours when vehicular activities are much. 

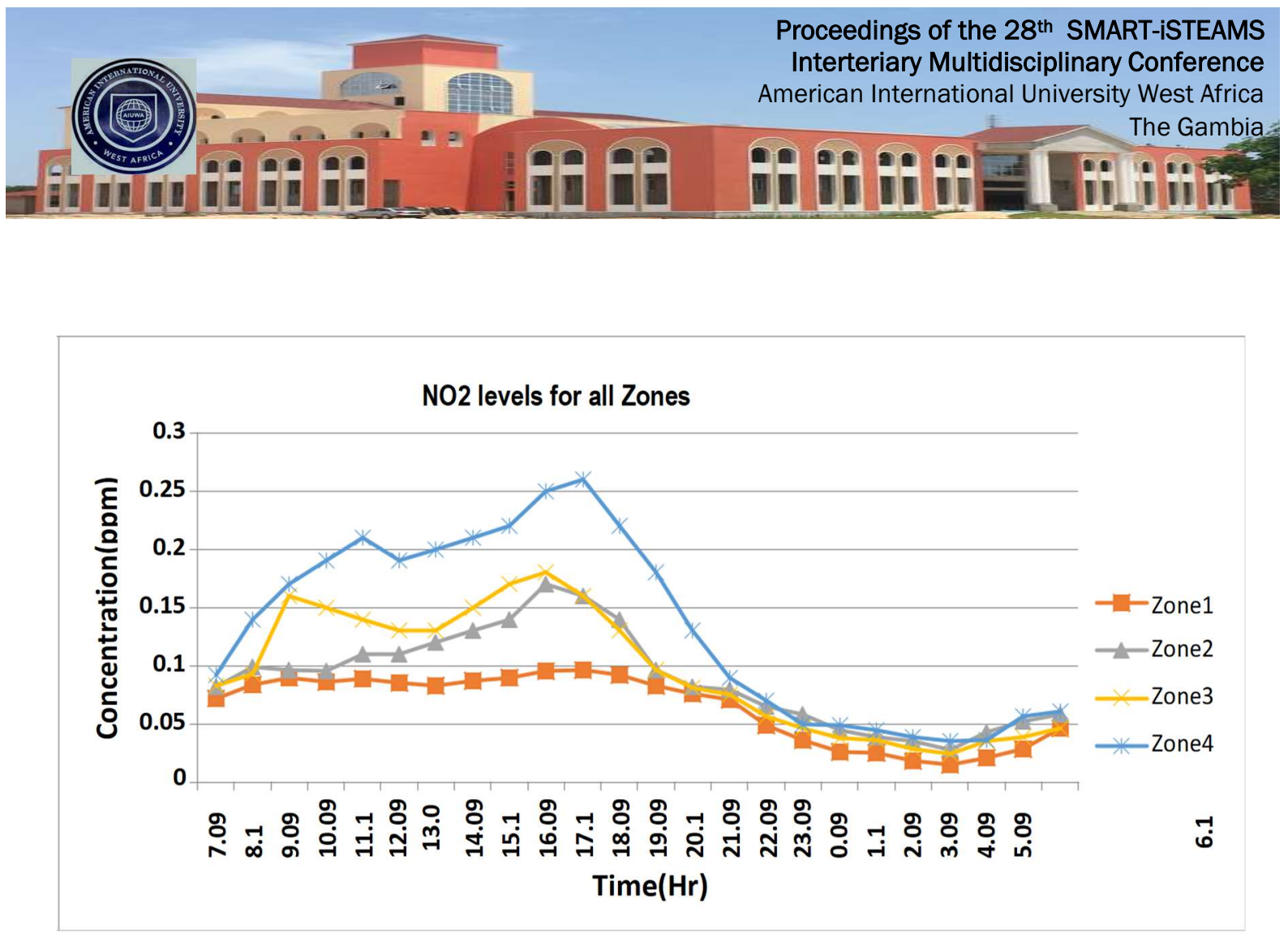

Fig 7. Chart of NO2 emission levels for Zone 1 to Zone 4

Zone 4 shows a significant level of NO2 when compared to other zones. The major source of the concentration levels being high is due to the high vehicular activities, from gasoline and diesel vehicles.

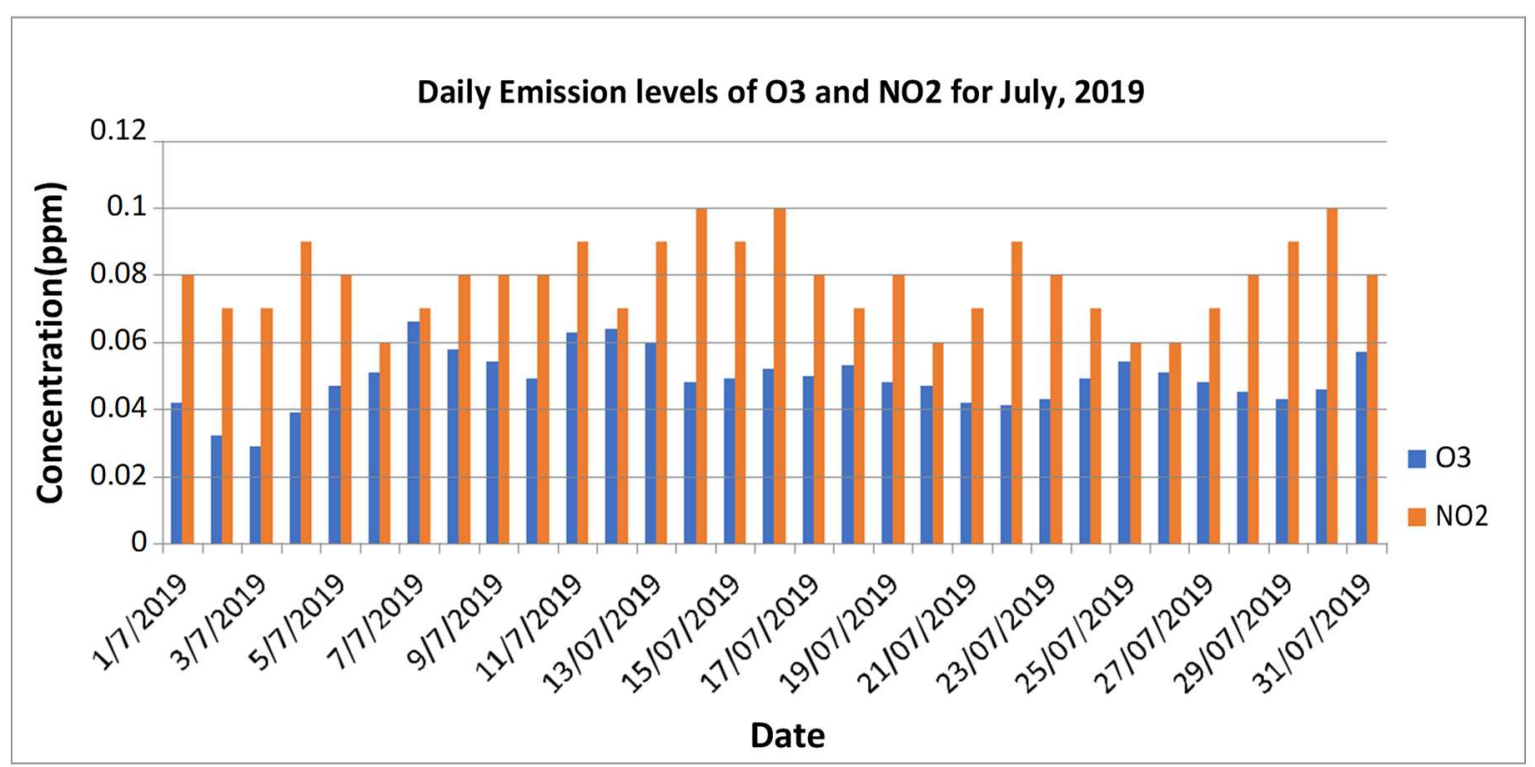

Fig 8: Daily 03 and NO2 emission level 


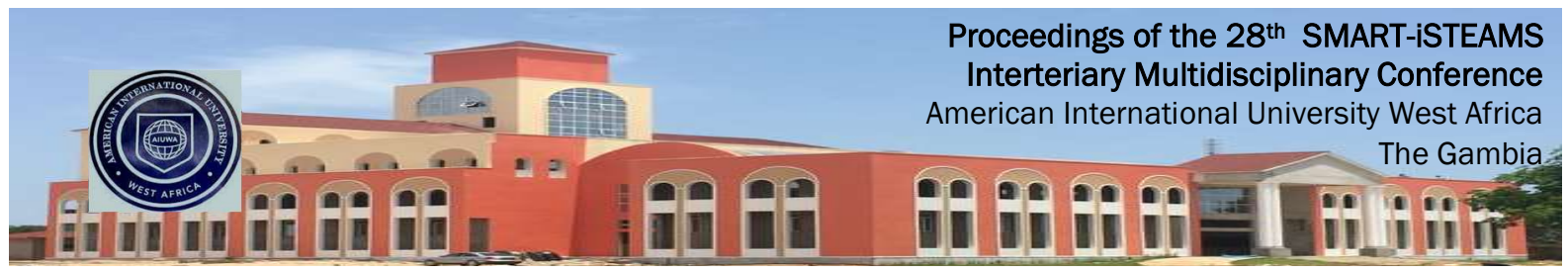

According to recommended standard for 03 is $0.05 p p m$ and $0.06 \mathrm{ppm}$ for NO 2 ( FEPA \& WHO). $\mathrm{O} 3$ level is poor as it swings from above and below thresholds and this shows there is potential danger of excess. NO2 exceeds the standard and as such prolong exposure to these levels there is tendency for sensitive individuals to suffer respiratory related illnesses.

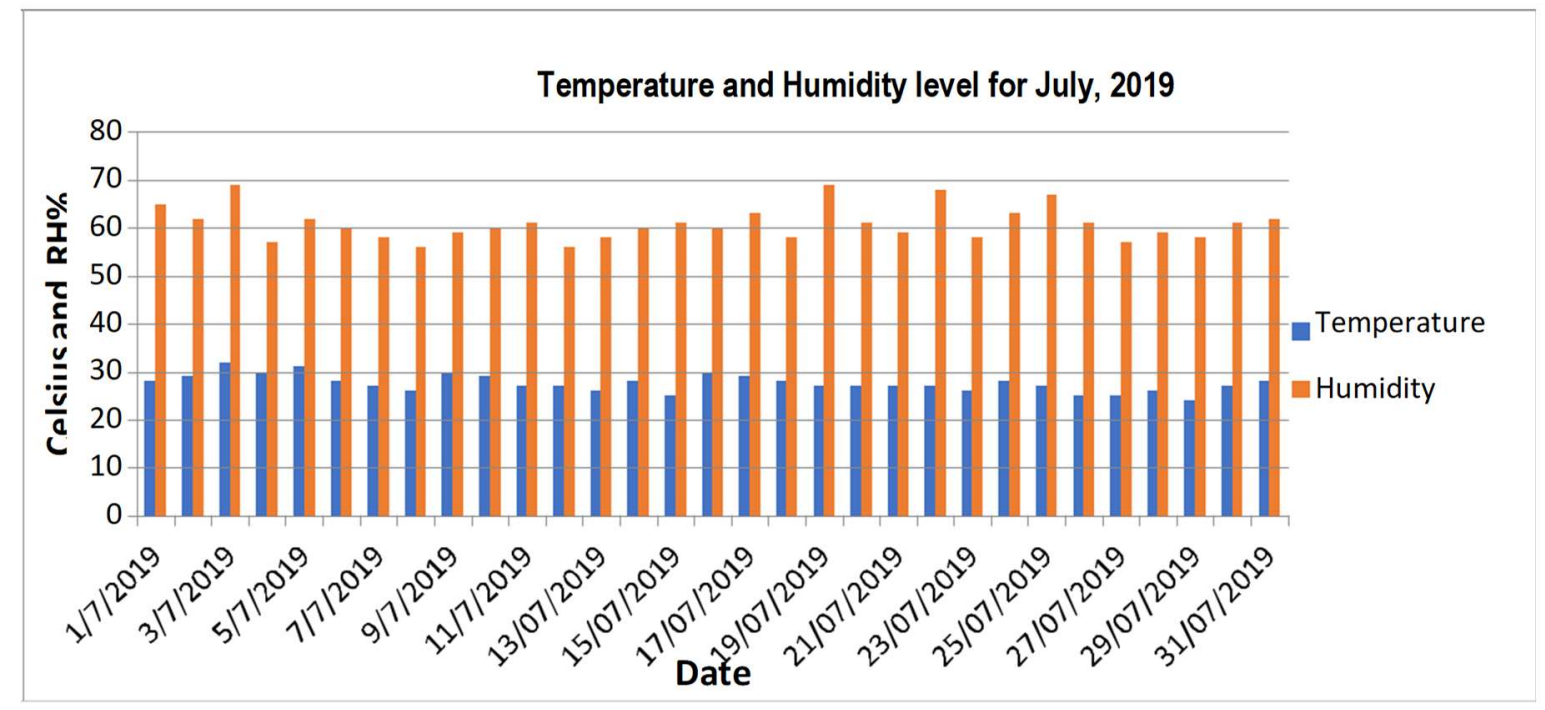

Fig 9. Daily Temperature and Humidity levels

TABLE 2: RESULTS FOR NO2 MONTHLY AVERAGES TREND FOR 2019

\begin{tabular}{|c|c|c|c|}
\hline Date & NO2 & Temperature & Humidity \\
\hline January & 0.078 & 28 & 61 \\
\hline February & 0.072 & 29 & 57 \\
\hline March & 0.069 & 30 & 53 \\
\hline April & 0.068 & 29 & 58 \\
\hline May & 0.069 & 29 & 59 \\
\hline June & 0.065 & 28 & 60 \\
\hline July & 0.061 & 27 & 59 \\
\hline August & 0.058 & 27 & 61 \\
\hline September & 0.057 & 28 & 60 \\
\hline October & 0.059 & 28 & 63 \\
\hline November & 0.060 & 29 & 58 \\
\hline December & 0.064 & 29 & 60 \\
\hline Yearly & & & 59.1 \\
\hline Average & 0.065 & 28.5 & \\
\hline
\end{tabular}




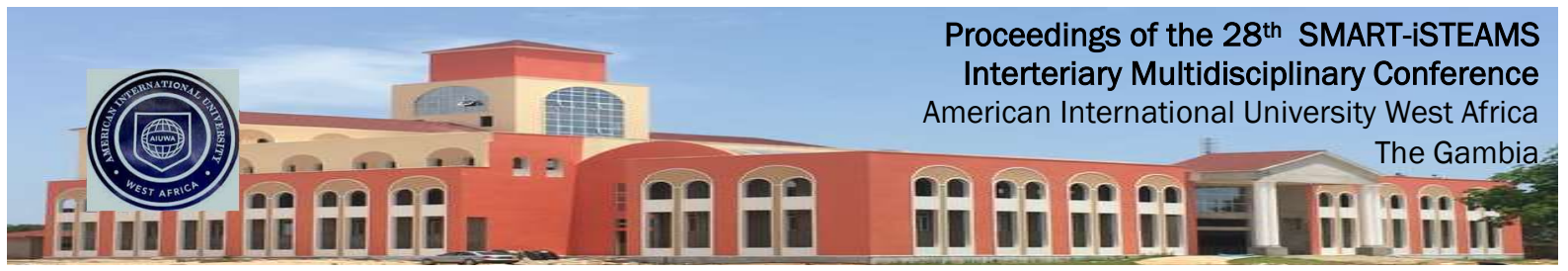

\section{CONCLUSION}

A real-time pollutant monitoring system was developed to monitor Nitrogen oxide gas concentration in the chosen area. This was implemented considering its importance and relevance in the present scenario. A detailed study and survey were done in terms of technical viability and economic feasibility in deploying monitoring systems in other industrial areas of the state. Experimentation was carried out at the industrial area of Ikeja, Lagos state and the developed wireless monitoring system was able to collect the air pollutant data consistently under the various conditions and timings.

\section{REFERENCES}

[1] H. Karl and A. Willig, "Protocols and Architectures for Wireless Sensor Networks", John Wiley and Sons Ltd, The Atrium, Southern Gate, Chichester, West Sussex, England, 2005

[2] W.A. Wardhana, "Impact of Environmental Pollution”, Andi Offset, Yogyakarta, 2004.

[3] Yanismai, "Relationship between traffic density and air quality in the Padang City". Padang, 2004.

[4] Tania, S. and Driejena S, " Roadside Concentration in the City of Bandung", Faculty of Civil Engineering Environmental Engineering Study Program, Institute of Technology, Bandung, 2009.

[5] M.G. Ghozikali, et al., "Quantification of the health effects of exposure to air pollution (NO2) in Tabriz, Iran," Fresenius Environmental Bulletin, vol. 24, no. 11c, pp. 4142-4148, 2015.

[6] WHO (World Health Organization), "Ambient Air Pollution: A global assessment of exposure and burden of disease", Geneva, 2016

[7] World Health Organization Fact sheet: " the top 10 causes of death": the 10 leading causes of death in the world, 2000 and 2011, Geneva, 2014. [online]. www.who.int.

[8] Purwadi, J. "Analysis of noise levels and emissions in slamet riyadi street and alternative solutions [Thesis]". Muhammadiyah University. Surakarta, 2006.

[9] Nihal Kularatna, and B. H. Sudantha, "An Environmental Air Pollution Monitoring System Based on the IEEE 1451 Standard for Low Cost Requirements" , IEEE Sensors Journal, Vol. 8, No. 4, 2008

[10] Young J.J, Yang K. L, Dong G.L, Keun H.R, S.Nittel , "Air pollution monitoring system based on geosensor network", IEEE International Geoscience and Remote Sensing Symposium, pp.III-1370III-1373, 2008

[11] Raja Vara Prasad Y, Mirza Sami Baig, Real Time Wireless Air Pollution Monitoring System, ICTACT Journal on Communication Technology: Special Issue on Next Generation Wireless Networks and Applications, June 2011, Volume: 2, Issue: 2

[12] Carles Araguz, Marc Marí, Elisenda Bou-Balust, Eduard Alarcon, and Daniel Selva. "Design Guidelines for General-Purpose Payload-Oriented Nanosatellite Software Architectures", Journal of Aerospace Information Systems, Vol. 15, No. 3 (2018), pp. 107-119

[13] C.K Akinsanya. "Recent trends in the pollution load on the Lagos Lagoon - Lagos State perspective". A paper presented on ecological sustainable industrial development workshop organized by UNIDO .2003.

[14] A. A. Oketola. "Industrial pollution assessment in Lagos, Nigeria using industrial pollution projection system and effluent Analysis". PHD thesis, Department of chemistry, university of Ibadan.2007.

[15] A.A Oketola and O Osibanjo. "Estimating sectoral pollution load in Lagos by Industrial pollution projection system (IPPS): Employment versus Output, Toxicological and Environmental chemistry": Pages 799-800.2009.

[16] MA. Abegunde. "Aspect of the physical environment of Lagos. In: Adefuye A, Agiri B, Osuntokun J, editors. History of the people of Lagos State. Lagos": Literamed Publications;1987. p. 6

[17] Sousan, S., Koehler, K., Hallett, L., and Peters, T. M.: "Evaluationof the Alphasense optical particle counter (OPC-N2) and the Grimm portable aerosol spectrometer" (PAS-1.108), Aerosol Sci.Tech., $50,1352-1365,2016$ 